\section{Escherichia coli 0157:h7 en carne molida comercializada en los mercados de Guayaquil}

Escherichia coli o157:h7 in ground beef commercialized in the markets of Guayaquil

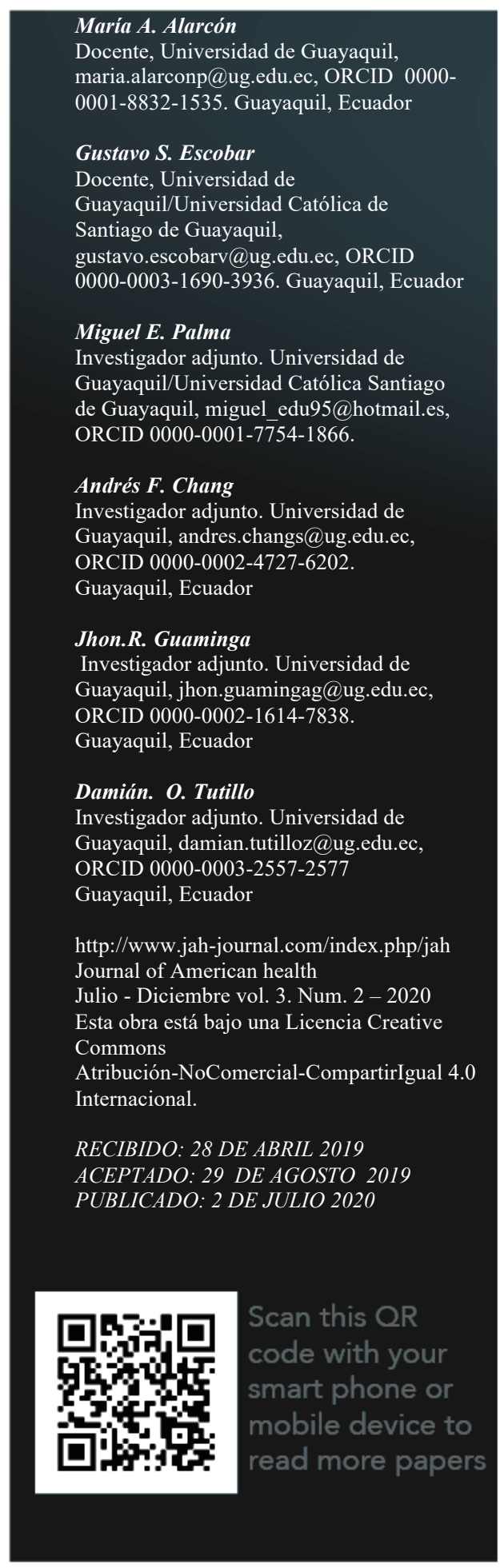

\section{RESUMEN}

Presencia de Escherichia coli $0157 \mathrm{H} 7$ a partir de carne molida de bovinos que se expende en los mercados de la Ciudad de Guayaquil. Metodología: El estudio fue de tipo descriptivo, observacional, con un diseño no experimental. Se analizaron un total de 200 muestras, enriquecidas en agua peptonada y cultivadas en agar rojo bilis neutro cristal violeta. Resultados: Las colonias identificadas como E. coli por pruebas bioquímicas, fueron serotipificadas para determinar la presencia de E. Coli 0157:H7. De las 200 muestras analizadas un $46.5 \%$ resultó positivas para $E$. coli y un 1.5\% para el serotipo 0157:H7. Conclusión: Demostrándose de acuerdo a la Norma INEM 1338:2012 la deficiente calidad microbiológica del alimento comercializado en la Ciudad de Guayaquil, lo que podría estar ocasionando riesgos de salud pública.

PALABRAS CLAVE: Escherichia coli, entero bacteria, toxina Shiga, serotipo 0157:H7, patógenos potenciales

\section{ABSTRACT}

The objective of this study was to determine the presence of Escherichia coli O157:H7 in ground beef sold in the markets of Guayaquil. The method applied was descriptive, observation and nonexperimental design. 200 samples were analyzed. All of then enriched with peptone water and then using violet red bile lactose agar. Colonies identified as E. coli were further identified as E. coli O157:H7. Results obtained were compared with the Ecuadorian INEM Standard 1338:2012 and showed that ground beef sold in the market of Guayaquil has poor microbiology quality with potential risk health. 
KEYWORDS: hopscotch game, psychomotricity, body scheme, laterality, balance.

\section{INTRODUCCIÓN}

Escherichia coli es una entero bacteria común en la flora bacteriana de humanos y animales. En determinadas circunstancias, estas bacterias presentan serios riesgos para la salud. Un problema emergente en enfermedades trasmitidas por alimentos lo constituye las variedades productoras de toxina Shiga (STEC) de las cuales el serotipo 0157:H7 es probablemente el más estudiado al momento (1). Otros serotipos considerados como patógenos potenciales y problema de salud pública por la Organización Mundial de la Salud (OMS) son O26:H11, 0103:H2, O111: NM, 0113:H21 y 0145: NM (2-3)Todas estas cepas presentan potencial para causar diarrea, colitis hemorrágica, síndrome urémico hemolítico (HUS por sus siglas en ingles) y daño renal $(4,5)$.Se ha estimado a nivel mundial que $E$. coli productora de toxina Shiga causa 2,801,00 enfermedades agudas anualmente (6).

\section{Frecuencia de detección en SA}

Las cepas de E. coli entero-hemolíticas han sido reportadas en todos los continentes (7). Investigaciones en Argentina, en donde la industria ganadera es una fuente importante de exportación, indicaron una frecuencia de $4.1 \%$ del serotipo 0157 en muestras de heces en bovinos y $2.6 \%$ en carcasas (8). Datos epidemiológicos en Brasil han detectado tanto el serotipo 0157 como los serotipos O26, 0103, 0111, 0118 en ganado vacuno (9). Serotipos productores de toxina Shiga diferentes al prototipo 0157 así como en Argentina (1) Se ha reportado una incidencia de HUS que alcanza a 400 casos/año (10). Estudios realizados en Lima - Perú de 195 muestras de carne molida de bovino analizadas el $87.18 \%$ fueron positivas para $E$. coli y de estas un $1.54 \%$ para E. coli $0157 \mathrm{H} 7$ (11).

En Ecuador no existen muchos estudios que demuestren el factor de virulencia de este microorganismo, (12) realizó un estudio en ganado doméstico en la ciudad de Quito, utilizando 600 muestras de hisopados rectales, encontrándose un 5\% positivo para E. coli O157:H7. (13) presenta un estudio de carnes de ganado vacuno, de 450 reses provenientes de la región sierra y región costa, con un 3\% de muestras positivas para E. coli 0157:H7.

A partir de la evidencia disponible, es probable que el control de cepas invasivas y patógenas de $E$. coli sea una prioridad en el control de la Salud en Ecuador $(14,15)$. 


\section{MATERIALES Y MÉTODOS}

Recolección de muestras:

Las muestras fueron recogidas y procesadas en los periodos del mes de octubre a diciembre del 2017 y noviembre a diciembre del 2018, en la cual se recolectaron 200 muestras distribuidas de la siguiente manera:

Mercado A, ubicado en la zona sur de la Ciudad de Guayaquil.

Mercado B, ubicado en la zona centro Oeste de la Ciudad de Guayaquil.

Mercado C, ubicado en la zona Norte de la Ciudad de Guayaquil.

De acuerdo a la Norma Técnica NTE INEM 776:2012 para el muestreo de carne molida y productos cárnicos, se procedió de la siguiente manera:

a recolección y transporte de las muestras de carne se realizó con todas las condiciones de esterilidad para evitar contaminación cruzada.

Las muestras fueron depositadas en fundas estériles con cierre hermético en una hielera Rubbermaid manteniendo una temperatura de $4-8^{\circ} \mathrm{C}$ para el transporte.

Las muestras cárnicas fueron procesadas dentro de las 2 horas desde la toma para asegurar resultados viables y confiables.

Fueron procesadas en el Laboratorio de Microbiología de Alimentos de la Facultad de Ciencias Químicas en la cual se procedió a pesar 10 gramos de la muestra, la misma que fue diluida en agua peptonada, para la respectiva siembra en agar rojo bilis cristal violeta, las colonias sospechosas para E. coli, fueron sembradas en TSI y luego realizadas las respectivas pruebas bioquímicas

Las muestras identificadas como E.coli, fueron preparadas para ser sometidas a la serotipificación para 0157:H7, mediante ensayo de aglutinación wellcolatex.

\section{RESULTADOS}

Ilustración 1: microorganismos aislados en las muestras de carne molida

\begin{tabular}{cccccc}
\hline Matriz & Grupo de & $\begin{array}{c}\text { Contaminación } \\
\text { nivel (UFC } \\
\text { total) }\end{array}$ & $\begin{array}{c}\text { Enriquecimiento } \\
\text { medio }\end{array}$ & $\begin{array}{c}\text { Tiempo de } \\
\text { incubación }\end{array}$ & $\begin{array}{c}\text { Presencia } \\
\text { (\%) }\end{array}$ \\
$\begin{array}{c}\text { (unidad de } \\
\text { muestra }\end{array}$ & - positivo & & \\
cantidad) & & réplicas / total & &
\end{tabular}

\begin{tabular}{llllll}
\hline $\begin{array}{l}\text { Carne } \\
\text { molida }\end{array}$ & $\begin{array}{l}\text { Klebsiella } \\
\text { pneumoniae }\end{array}$ & ----- & Agua de pectona & 48horas & $16.5 \%$ \\
& & & &
\end{tabular}

(10 g) 


\begin{tabular}{|c|c|c|c|c|}
\hline $\begin{array}{l}\text { Klebsiella } \\
\text { oxytoca }\end{array}$ & ------ & Agua de pectona & 48 horas & $15,5 \%$ \\
\hline $\begin{array}{l}\text { Proteus } \\
\text { mirabilis }\end{array}$ & ------ & Agua de pectona & 48 horas & $14,5 \%$ \\
\hline $\begin{array}{l}\text { Providencia } \\
\text { morgani }\end{array}$ & ------ & Agua de pectona & 48 horas & $7 \%$ \\
\hline $\begin{array}{l}\text { Escherichia } \\
\text { coli }\end{array}$ & $\begin{array}{l}\text { Mayor a } 10^{2} \\
\text { UFC/g muestra }\end{array}$ & Agua de pectona & 48 horas & $46,5 \%$ \\
\hline
\end{tabular}

De acuerdo a lo observado en la imagen \# 1. Un 53.5\% de las muestras analizadas, refleja la presencia de otros microorganismos pertenecientes a la familia de las enterobacterias. En relación a la presencia de $E$. coli en carne molida un $46.5 \%$ fueron positivas para $E$. coli, microorganismos indicadores de contaminación fecal, siendo los conteos, superiores a lo permitido por la norma INEM 1346: 2016. Resultados que se relacionan con la carencia de Buenas Practicas de higiene en el proceso.

\section{Ilustración \# 2. Análisis para E. coli 0157:H7}

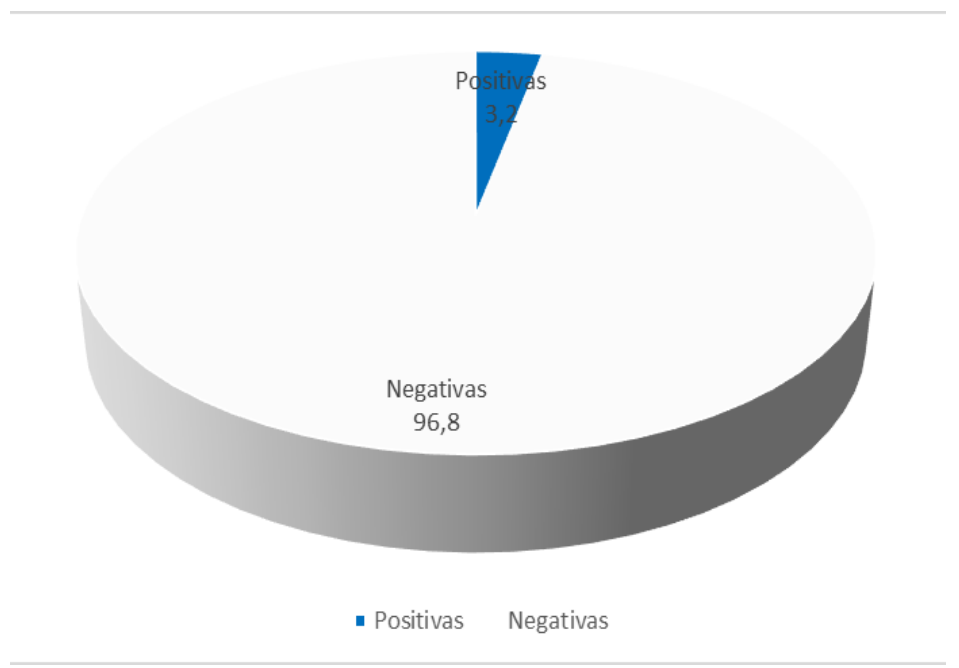

En la imagen \# 2 de acuerdo a la serología de las muestras positivas para E. coli un $3.2 \%$ dieron positivas para E. coli O157:H7.

La Norma INEM 1346:2016 estable los requisitos que deben cumplir la carne y productos cárnicos con la finalidad de prevenir los riesgos para la salud y la vida de las personas y evitar prácticas que puedan inducir a error a los usuarios, permite un conteo de $E$. coli de $1 \times 10^{1} \mathrm{ufc} / \mathrm{g}$ 
y ausencia para E. coli 0157:H7. Demostrando de esta manera que el producto se encuentra no apto para el consumo humano.

Ilustración 3. Evaluación de las condiciones de la temperatura en el lugar de expendio de la carne molida en los mercados de estudio

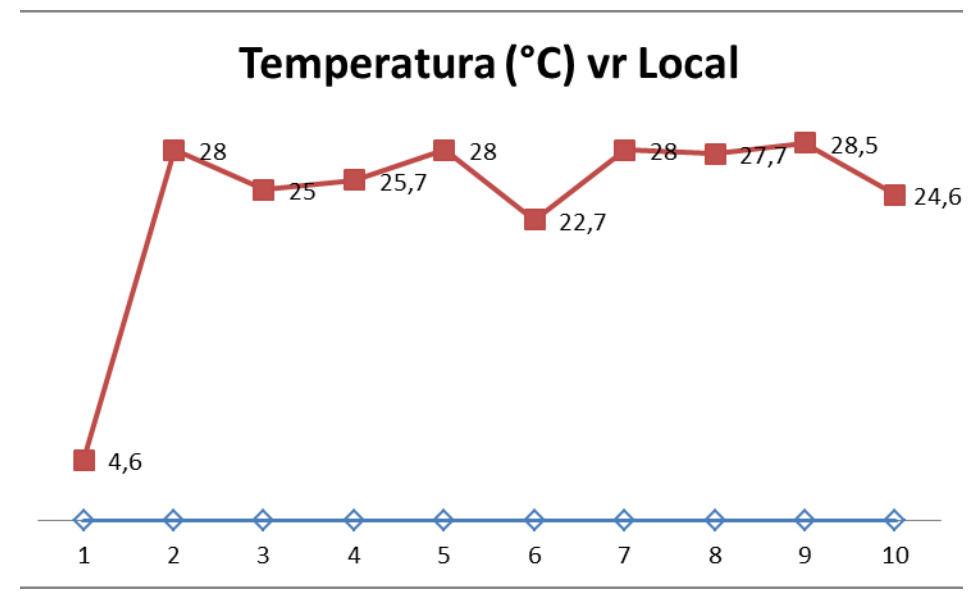

Se pudo observar que de los 10 locales muestreados las condiciones de venta de las carnes en 9 locales, se lo efectúa a temperatura ambiente $\left(22,7^{\circ} \mathrm{C}-28,5^{\circ} \mathrm{C}\right)$ y solo un local conservaba las muestras en refrigeración $\left(4,6^{\circ} \mathrm{C}\right)$. Según el INAMHI las temperaturas promedias para el momento de la toma de muestras (condiciones meteorológicas Guayaquil-Durán) registraron un mínimo de $22,0^{\circ} \mathrm{C}$ y un máximo de $32^{\circ} \mathrm{C}$.

\section{DISCUSIÓN}

A nivel mundial, la carne molida se considera el reservorio principal de E. coli O157:H7, productor de una infección asociada con brotes de colitis hemorrágica y el síndrome urémico hemolítico (17). En Ecuador existen muy pocos estudios que demuestren la presencia de este agente patógeno que es causante de algunos cuadros clínicos. Los casos reportados hasta el momento han sido identificados por serotipificación (Wellcolatex), indicando que la cepa se encuentra presente en los productos cárnicos que se comercializan en los mercados del país. Por lo tanto, se hace necesario el control riguroso de medidas higiénicas en los rastros del país, así como desarrollar programas sobre la importancia de la correcta cocción de los alimentos e instruir al manipulador la aplicación de las Buenas Prácticas de manipulación, para mejorar la calidad microbiológica del alimento. 
Así también se hace necesario continuar los estudios de caracterización molecular de las cepas de $E$. coli productoras de la toxina Shiga (STEC) para determinar los genes que codifican la toxina que son importantes factores de virulencia en la patogenia de la enfermedad.

\section{CONCLUSIONES}

Los resultados obtenidos del presente trabajo de investigación son comparables a los obtenidos en Argentina en que, de 169 muestras de carne molida de bovino, tomada de diferentes mercados y analizadas, un $5.32 \%$ fueron positivas para $E$. coli 0157:H7 y en un segundo estudio en el 2015 reporto un 7.6\% muestras positivas para E. coli O157:H7 (16).

En relación a la evaluación de las condiciones higiénicas en los puestos de venta, se realizó un control de temperatura de los puestos de los Mercados A - B - C de la Ciudad de Guayaquil Ecuador, lo que indicó que, de los 10 locales un 7\% cumplían con las condiciones de temperatura requerida en sus contenedores de almacenamiento $\left(4,6^{\circ} \mathrm{C}\right)$, mientras que un $93 \%$ mantuvieron la carne molida a temperatura ambiente (temperatura ambiente media $21^{\circ} \mathrm{C}-$ $31^{\circ} \mathrm{C}$. Por lo tanto: el producto cárnico al estar expuesto a temperatura ambiente, acelera la proliferación bacteriana.

En la evaluación de la condición higiénica en los puestos de venta de carne molida se evidencio que un $31 \%$ presentaba condiciones higiénicas muy buena de acuerdo a las normas que dicta el Manual del manipulador de alimentos (FAO) un $60 \%$ una condición higiénica relativamente buena y un $9 \%$ de los locales presentó condiciones higiénicas bastantes deficientes, y de acuerdo a estas condiciones en estos puestos la presencia de E. coli / E. coli O157:H7 fue de un $46,5 \%$ (Tabla 1).

\section{REFERENCIAS}

1. Pérez Terrazzinoa, GB. Condori, MS. López Campo, A., Vega, S. Carbonari, C., Chinene, I. Rivas, M. de Castilloa MC., Jure MA. Calidad higiénico-sanitaria en plantas de faena de la provincia de Tucumán. Detección, aislamiento y caracterización de Escherichia coli productor de toxina Shiga. Revista Argentina de Microbiología.2017 49(3).242-246

2. FAO. Evaluación del riesgo de Escherichia coli enterohemorrágica (STEC) en los productos de carne fresca y carne de res. 2011.http://www.fao.org/food/food-safety-quality/a-zindex/e-coli/es/ 
3. Brusa V, Aliverti V, Aliverti F, Ortega EE, De la Torre JH, Linares LH, Sanz ME. Etcheverría, Al. et al. Shiga toxin-producing Escherichia coli in beef retail markets from Argentina. Front Cell Infect Microbiol. 2013 2.171. doi: 10.3389/fcimb.2012.00171

4. Farfán A, Ariza S, Vargas F,Vargas V. Mecanismo de virulencia de Escherichia coli enteropatogena.Rev. Chiena Infectol. $2016 \quad$ 33(4):438-450. https://scielo.conicyt.cl/pdf/rci/v33n4/art09.pdf

5. Varela, SchelottoF. Síndrome urémico hemolítico en Uruguay. Aspectos microbiológicos y clínicos,aportes para su conocimiento regional.Revista Salud UDES. 2015. file://C:/Users/USUARIO/Downloads/26-44-1-SM\%20(1).pdf

6. Amagliania G, Rotundoa L, Carlonia E, Omicciolic E, Magnania M, Giorgio Brandia G, Fratamicob. Detection of Shiga toxin-producing Escherichia coli (STEC) in ground beef and bean sprouts: Evaluation of culture enrichment conditions. Food Research International. 2018. 103, 398-405. https://doi.org/10.1016/j.foodres.2017.10.059

7. Institute for international cooperation in Animal Biologics. Escherichia coli Productora de Toxina Shiga (STEC), Escherichia coli 0157:H7.The Center for Food Security y Public Health. 2009.http://www.cfsph.iastate.edu/Factsheets/es/ecoli-es.pdf

8. Del Castillo L, Masana M, Leotta G. Detección y caracterización de Escherichia coli o157 de ganado bovino faenado en frigoríficos de la Argentina. Universidad Nacional de la Plata, $\begin{array}{llll}\text { Facultad de } & \text { Ciencias }\end{array}$ http://sedici.unlp.edu.ar/bitstream/handle/10915/45222/Documento completo.pdf?seq uence $=3 \&$ is Allowed $=y$

9. Instituto Nacional de Salud, Grupo de Evaluación de Riesgos en Inocuidad de Alimentos. Perfil de riesgo de Escherichia coli enterotoxigénica y verotoxigénica en queso fresco. $\begin{array}{llll}\text { Bogotá, } & \text { D. } & \text { C., } & \text { Colombia. }\end{array}$ https://www.ins.gov.co/Direcciones/Vigilancia/Publicaciones\%20ERIA\%20y\%20Plaguicid as/PERFIL\%20E.\%20COLI.pdf

10. SENASA.Síndrome urémico hemolítico: pequeños grandes cuidados. 2017 http://www.senasa.gob.ar/senasa-comunica/infografias/sindrome-uremico-hemoliticopequenos-grandes-cuidados 
11. Méndez CR, Vergaray G , Morante HY, Flores, PR \& Gamboa RA. Isolation and characterization of Escherichia coli 0157:H7 from ground beef cattle in Lima-Peru. Rev. Peru. biol. 2013 20(2): 159 - 164

12. Trueba G, Garcés V, Barragan V, Colman R.E, Seymour M, Vogler A.J, Keim, P. Escherichia coli 0157:H7 in Ecuador: animal reservoirs, yet no human disease. Vector Borne Zoonotic 2013. Dis. 13:295-8.

13. Hidalgo A, Bastidas, A. Determinación de Escherichia coli 0157: H7 por el método Oficial AOAC 996.09 en carne de res faenada, proveniente de la empresa metropolitana de rastro de Quito. Repositorio digital Universidad central del Ecuador. 2018 http://www.dspace.uce.edu.ec/handle/25000/17604

14. Bhavnani D, Bayas R de L, Lopez V.K, Zhang L, Trueba G, Foxman B, Marrs C, Cevallos W, Eisenberg, J.N. Distribution of Enteroinvasive and Enterotoxigenic Escherichia coli across Space and Time in Northwestern Ecuador. Am J Trop Med Hyg. 2016 94:276-84.

15. Rao G, Eisenberg J.N.S, Kleinbaum D.G, Cevallos W, Trueba G, Levy K. Spatial Variability of Escherichia coli in Rivers of Northern Coastal Ecuador. Waters 2015. 7: 818-832.

16. Jure $M$, Condori M, Pérez P, Terrazzino G, López Campo A, Zolezzi G, Chinen, I, Rivas M, Catillo M. Aislamiento y caracterización de $E$ coli 0157 en productos cárnicos bovinos y medias reses en la provincia de Tucumán. Rev Argent Microbiol. 2015 47:125-131.

17. 17. Mendez C, Vergaray G, Morante H, Flores G, Gamboa R.Aislamiento y caracterización de Escherichia coli 0157:H7 a partir de carne molida de bovino en Lima-Peru. Revista Peruana de Biologia. 2013 20:159-164.

18. Ardissino G, Salardi S, Colombo E, Testa S, Borsa-Ghiringhelli N, Paglialonga F, Paracchini $\mathrm{V}$, Tel F. Epidemiology of haemolytic uremic syndrome in children. Data from the North Italian HUS network. Eur J Pediatr. 2016 175:465-73.

19. Banatvala N, Griffin P.M, Greene K.D, Barrett T.J, Bibb W.F, Green J.H, Wells J.G, and the Hemolytic Uremic Syndrome Study Collaborators. The United States National Prospective Hemolytic Uremic Syndrome Study: microbiologic, serologic, clinical, and epidemiologic findings. J Infect Dis. 2001 183:1063-70. 
20. Blanco J.E, Blanco M, Blanco J, Escribano A. Escherichia coli enterotoxigénica, verotoxigenicas y necrotoxigénicas en alimentos y muestras clínicas. Papel de los animales como reservorio de cepas patógenas para el hombre. Microbiología SEM. 1995 11:97-110.

21. Boerlin P, McEwen S.A, Boerlin-Petzold F, Wilson, J.B, Johnson, R.P, Gyles C.L. Associations between virulence factors of Shiga toxin-producing Escherichia coli and disease in humans. 1999) J Clin Microbiol. 37:497-503.

22. Briones M, Tapia F. Determinación de Escherichia Coli 0157:H7 (EHEC) en la carne molida que se vende en el mercado el Arenal de la ciudad de Cuenca. Tesis, Universidad del Azuay. 2016 http://dspace.uazuay.edu.ec/handle/datos/6315.

23. FAO: Food and Agriculture Organization of the United Nations.Livestock Sector Brief: $\begin{array}{llll}\text { Ecuador. Food and Agriculture Organization, Rome. } 2002 & \end{array}$ www.fao.org/ag/againfo/resources/en/publications/sector briefs/lsb ECU.pdf

24. Page A.V, Liles W.C.Enterohemorrhagic Escherichia coli Infections and the HemolyticUremic Syndrome. Med Clin North Am. 2013 97:681-95.

25. Palmeira P, Carbonare S.B, Amaral J.A, Tino-De-Franco, $M$, et al. Colostrum from healthy Brazilian women inhibits adhesion and contains IgA antibodies reactive with Shiga toxin producing Escherichia coli. Eur J Pediatr. 2005 164:37-43.

26. Parsons B.D, Zelyas N, Berenger B.M , Chui L. Detection, Characterization, and Typing of Shiga Toxin-Producing Escherichia coli. Front Microbiol. 2016 12:478.

27. Perez J.C. En ocho provincias se concentra el mayor consumo de cárnicos. www.revistalideres.ec/lideres/consumo-carnicos-ecuador.html 2015.

28. Rivero M, Passucci J, Lucchesi P, Signorini M., Alconcher L, Rodriguez E, Rocha V, Meneguzzi, B.Epidemiologia del Sindrome urémico hemolítico en dos regiones de la provincia de Buenos Aires. Medicina 2013 73:127-135.

29. Rodríguez Á.G. Principales características y diagnóstico de los grupos patógenos de Escherichia coli. Salud Pública de México. 2012 44, 464-475. 
30. Smith J.L, Fratamico P.M, Gunther N.W. $4^{\text {th }}$. Shiga toxin-producing Escherichia coli. Appl Microbiol. . 2014 86:145-97.

31. Stenkamp-Strahm C, McConnel C, Magzamen S, Abdo Z, \& Reynolds S. Associations between Escherichia coli $\mathrm{O} 157$ shedding and the faecal microbiota of dairy cows. J Appl Microbiol. 2018 124:881-898.

32. Tanaro J.D, Pianciola L.A, D'Astek B.A, Piaggio M.C, Mazzeo M.L, Zolezzi G. \& Rivas M. Virulence profile of Escherichia coli 0157 strains isolated from surface water in cattle breeding areas. Lett Appl Microbiol. 2018 Mar 3, 2018. doi: 10.1111/lam.12873. [Epub ahead of print].

33. Tanaro J.D, Piaggio M.C, Gasparovic A.M, Badaracco V.A, Tesouro R, Kesselman D, Indart, N.S, De Gracia, L. Escherichia coli 0157:H7 Productor de toxina Shiga aisladas de muestras de agua relacionadas a establecimientos pecuarios de engorde a corral. Ciencia, Docencia y Tecnología. et al. 2016 6: 138-155.

34. Xu T, Marr E, Lam, H, Ripp, S, Sayler, G., Close, D. Real-time toxicity and metabolic activity tracking of human cells exposed to Escherichia coli 0157:H7 in a mixed consortia. Ecotoxicology. 2015 24:2133-40.

35. Yan R, Liu Y, Gurtler J.B, Killinger K, Fan X. Sensitivity of pathogenic and attenuated E. coli O157:H7 strains to ultraviolet-C light as assessed by conventional plating methods and ethidium monoazide-PCR. J of Food Safety. 2017 doi: 10.1111/jfs.12346.

36. Organización de las Naciones Unidas para la Alimentación y la Agricultura y Organización Panamericana de la Salud / Organización Mundial de la Salud Washington, D.C.,CONDICIONES METEOROLOGICAS GUAYAQUIL - DURAN, www.serviciometeorologico.gob.ec > bolhist > DIARIO > NOVIEMBRE (2017) 
http://www.jah-journal.com/index.php/jah 